\title{
A arte e a ciência de escrever um artigo científico de revisão
}

Edison Iglesias de Oliveira Vidal 1

Fernanda Bono Fukushima 1

doi: 10.1590/0102-311X00063121

Artigos de revisão foram e permanecem recursos de grande valor nos diferentes campos da ciência. No entanto, o panorama dos artigos de revisão da literatura passou por grandes mudanças ao longo dos últimos 50 anos. Enquanto os artigos de revisão em Medicina e Saúde Pública na era anterior ao advento da medicina baseada em evidências eram quase que exclusivamente de natureza não sistemática 1, os anos 1990 e o novo milênio testemunharam a ascensão do campo da síntese de pesquisas como uma disciplina científica própria 2,3. Por exemplo, em 1999 cerca de 300 referências relativas a revisões sistemáticas foram indexadas no PubMed, enquanto que 20 anos depois, em 2019, mais de 27 mil referências de revisões sistemáticas foram adicionadas àquela base. Não apenas o número de revisões cresceu exponencialmente, a complexidade e diversidade dos métodos de revisão também passaram por um progresso tão importante que é frequentemente impossível a um único pesquisador acompanhar o passo dessa evolução e dominar todos os avanços desse campo efervescente 4,5,6,7.

Mas por que boas revisões de literatura são tão importantes? John W. Strutt (Lord Rayleigh), vencedor do Prêmio Nobel de Física de 1905, em seu discurso presidencial para a Associação Britânica para o Avanço da Ciência em 1884, argumentou que o empreendimento científico é um processo que consiste ao mesmo tempo na produção de conhecimentos novos e de sua integração com o antigo ${ }^{8}$. Defendeu ainda que se a ciência consistisse apenas no acúmulo de fatos, que ela seria esmagada por seu próprio peso. Nesse âmbito, as revisões da literatura desempenham um papel essencial através da integração judiciosa dos conhecimentos novos e velhos. Adicionalmente, as revisões são valiosas por fornecer contexto histórico, apontar lacunas no conhecimento existente e caminhos futuros a serem percorridos 9. Cada vez mais, um fator importante para a ascensão do campo da síntese de pesquisas decorre diretamente do fato de que revisões de alta qualidade têm sido demandadas e consumidas não apenas por pesquisadores e profissionais de saúde, mas também pelos responsáveis pelo desenvolvimento de políticas de saúde e pelo público de forma geral 3 . De fato, as Colaborações Cochrane (The Cochrane Collaboration. http://www.cochrane. org, acessado em 07/Mar/2021) e Campbell (The Campbell Collaboration. https://www. campbellcollaboration.org/, acessado em 07/Mar/2021) oferecem resumos em lingua-
1 Faculdade de Medicina de Botucatu, Universidade Estadual Paulista Júlio de Mesquita Filho, Botucatu, Brasil. 
gem leiga dos resultados das suas sínteses de pesquisa em saúde e ciências sociais para o público geral.

Tendo em vista a complexidade crescente dos métodos de revisão, que conselho pode ser dado a pesquisadores novatos considerando escrever um artigo de revisão? Nosso primeiro conselho envolve obter clareza sobre os objetivos da revisão. Os pesquisadores devem se perguntar por que a revisão é necessária e qual público pretendem alcançar. Uma compreensão clara dos objetivos da revisão frequentemente sinalizará os métodos mais apropriados para alcançá-los. Por exemplo, se os pesquisadores possuem uma pergunta com enfoque sobre a eficácia/efetividade de determinada prática ou tratamento sobre um conjunto de desfechos, ou pretendem que sua síntese influencie diretrizes clínicas ou políticas de saúde, então uma revisão sistemática seria o método de escolha. É importante ressaltar que existem ao menos 10 tipos de revisões sistemáticas, abrangendo desde avaliações econômicas até as metassínteses qualitativas ${ }^{6}$. Caso pretendam compreender a extensão e natureza da literatura sobre um dado assunto para determinar o valor e escopo de futuras revisões sistemáticas ou lacunas na literatura, então uma revisão de escopo provavelmente representaria a melhor abordagem para atender suas necessidades 10 . Por outro lado, caso os pesquisadores almejem fornecer uma perspectiva abrangente sobre determinado tema, desde sua história a avanços recentes, padrões atuais, controvérsias e caminhos futuros, então uma revisão narrativa seria provavelmente o melhor caminho a trilhar 11.

Nosso segundo conselho envolve tornar-se bastante íntimo dos princípios e métodos fundamentais do tipo de revisão escolhido e compor uma equipe para a revisão cujas expertises e dedicação seriam suficientes para endereçar seus principais desafios. Por exemplo, familiarizar-se com os métodos de revisões sistemáticas de intervenções demanda uma boa compreensão dos conteúdos dos manuais da Colaboração Cochrane 12 e do GRADE 13, das diretrizes PRISMA (http://prisma-statement.org/, acessado em 07/Mar/2021) e do instrumento AMSTAR 2 14. Para revisões de escopo, os autores devem estar familiarizados com o trabalho seminal de Arksey \& O’Malley 15 e desenvolvimentos recentes 10,16,17. Embora há pouco tempo tenha sido desenvolvida uma escala para avaliar a qualidade de revisões narrativas 18 , tal instrumento ainda se encontra distante do ideal. Portanto, para autores interessados em redigir uma revisão narrativa, aconselhamos um exame detalhado da estrutura das revisões narrativas publicadas na série Estado da Arte 19 do British Medical Journal, as quais fornecem um panorama amplo sobre um tema principal na área da saúde e ao mesmo tempo procuram se basear tanto quanto possível no resultado de revisões sistemáticas prévias. Idealmente, a equipe da revisão deve incluir ao menos um especialista no tema da revisão e outro em seus métodos específicos.

Nosso último conselho diz respeito ao que não fazer em um artigo de revisão. Primeiramente, deve-se evitar a todo custo padrões cansativos de apresentação de dados onde são produzidas listas extensas de afirmações de que "fulano et al." encontram X, "ciclana et al." demonstraram Y, enquanto "outrem et al." acharam Z. Esse tipo de apresentação dos resultados não conduz a qualquer síntese palpável e facilmente se torna uma fonte de desânimo para o leitor. Em segundo lugar, é importante evitar conclusões que não levem em consideração a complexidade do assunto em questão, como quando são realizadas afirmativas causais desconsiderando o grau de (in)certeza ou a qualidade das evidências. Em terceiro lugar, para revisões sistemáticas, onde a avaliação do risco de viés é obrigatória, é importante evitar o uso de instrumentos como as escalas de Newcastle-Ottawa e de Downs \& Black, que geram escores numéricos como parâmetro final para avaliação do risco de 
viés nos estudos incluídos. Embora comumente utilizadas, esse tipo de instrumento frequentemente falha ao identificar estudos com elevado risco de viés quando um único ou poucos domínios fundamentais estão gravemente comprometidos nos estudos incluídos na revisão 20,21. Atualmente estão disponíveis diversos instrumentos que evitam a ilusão de objetividade sugerida por escores numéricos para avaliar o risco de viés. Tais instrumentos requerem dos autores uma reflexão cuidadosa sobre questões importantes relacionadas a fontes de confundimento dentro do arcabouço causal de interesse para sua revisão (Risk of bias tools. https://sites.google.com/site/riskofbiastool/, acessado em 08/Mar/2021) 22. Em quarto lugar, os autores de revisões devem evitar o uso excessivo de abreviações e jargões que são habituais apenas para aqueles já experientes dentro de um campo específico do conhecimento, porque as revisões devem ser úteis para uma audiência mais ampla, incluindo aqueles que estão se aproximando do tema pela primeira vez ${ }^{9}$. Finalmente, recomendamos enfaticamente que não se tente disfarçar os capítulos introdutórios de teses ou dissertações como artigos de revisão. Embora seja esperado que tais capítulos introdutórios apresentem uma revisão da literatura, eles possuem objetivos, público alvo e grau de rigor diferentes daqueles esperados para um bom artigo de revisão.

Em conclusão, bons artigos de revisão habitam o coração do empreendimento científico. O ofício de escrever tais artigos envolve buscar alcançar o equilíbrio entre o rigor necessário para selecionar e avaliar criticamente estudos originais, e a arte de contar uma história ao fornecer contexto, explorar o conhecido e o desconhecido, e sinalizar o caminho à frente.

\section{Colaboradores}

E. I. O. Vidal contribuiu na concepção do artigo, redação do primeiro rascunho do manuscrito e aprovação da versão final. F. B. Fukushima contribuiu na revisão crítica do manuscrito, aprovação da versão final. Ambos autores concordam em assumir responsabilidade pelo conteúdo do mesmo.

\section{Informações adicionais}

ORCID: Edison Iglesias de Oliveira Vidal (00000002-1573-4678); Fernanda Bono Fukushima (0000-0001-8055-5425).
1. Mulrow CD. The medical review article: state of the science. Ann Intern Med 1987; 106:4858.

2. Grant MJ, Booth A. A typology of reviews: an analysis of 14 review types and associated methodologies. Health Info Libr J 2009; 26:91108.

3. Chalmers I, Hedges LV, Cooper H. A brief history of research synthesis. Eval Health Prof 2002; 25:12-37.

4. Nakagawa $S$, Samarasinghe G, Haddaway NR, Westgate MJ, O'Dea RE, Noble DWA, et al. Research weaving: visualizing the future of research synthesis. EcoEvoRxiv 2018; 10 nov. https://ecoevorxiv.org/ga2qz/ (acessado em 07/Mar/2021).

5. Cacchione PZ. The evolving methodology of scoping reviews. Clin Nurs Res 2016; 25:115-9. 
6. Munn Z, Stern C, Aromataris E, Lockwood C, Jordan Z. What kind of systematic review should I conduct? A proposed typology and guidance for systematic reviewers in the medical and health sciences. BMC Med Res Methodol 2018; 18:5.

7. Gough D, Thomas J, Oliver S. Clarifying differences between review designs and methods. Syst Rev 2012; 1:28.

8. Lord Rayleigh. Report of the fifty-fourth meeting of the British Association for the Advancement of Science [microform]: held at Montreal in August and September 1884. https:// archive.org/details/cihm_05877/page/n7/ mode/2up?q=Rayleigh (acessado em $07 /$ Mar/2021).

9. Tay A. How to write a superb literature review. Nature 2020; [Online ahead of print].

10. Peters MDJ, Godfrey CM, Khalil H, McInerney P, Parker D, Soares CB. Guidance for conducting systematic scoping reviews. Int J Evid Based Healthc 2015; 13:141-6.

11. Gregory AT, Denniss AR. An introduction to writing narrative and systematic reviews tasks, tips and traps for aspiring authors. Heart Lung Circ 2018; 27:893-8.

12. Higgins JPT, Thomas J, Chandler J, Cumpston M, Li T, Page MJ, et al. Cochrane handbook for systematic reviews of interventions. 2nd $\mathrm{Ed}$. Hoboken: Wiley-Blackwell; 2020. [Cochrane Book Series].

13. Schünemann H, Brozek J, Guyatt G, Oxman A. GRADE handbook. https://gdt.gradepro.org/ app/handbook/handbook.html (acessado em 07/Mar/2021).
14. Shea BJ, Reeves BC, Wells G, Thuku M, Hamel C, Moran J, et al. AMSTAR 2: a critical appraisal tool for systematic reviews that include randomised or non-randomised studies of healthcare interventions, or both. BMJ 2017; 358:j4008.

15. Arksey H, O’Malley L. Scoping studies: towards a methodological framework. Int J Soc Res Methodol 2005; 8:19-32.

16. Tricco AC, Lillie E, Zarin W, O’Brien KK, Colquhoun H, Levac D, et al. PRISMA Extension for Scoping Reviews (PRISMA-ScR): checklist and Explanation. Ann Intern Med 2018; 169:467.

17. Levac D, Colquhoun H, O'Brien KK. Scoping studies: advancing the methodology. Implement Sci 2010; 5:69.

18. Baethge C, Goldbeck-Wood S, Mertens S. SANRA - a scale for the quality assessment of narrative review articles. Res Integr Peer Rev 2019 26; 4:5.

19. The BMJ. State of the Art. Latest from the BMJ. https://www.bmj.com/specialties/state-art (acessado em 07/Mar/2021).

20. Vidal EIO. O que esperamos das revisões sistemáticas no futuro. Cad Saúde Pública 2016; 32:eED010916.

21. O'Connor SR, Tully MA, Ryan B, Bradley JM, Baxter GD, McDonough SM. Failure of a numerical quality assessment scale to identify potential risk of bias in a systematic review: a comparison study. BMC Res Notes 2015; 8:224.

22. Joanna Briggs Institute. Critical appraisal tools. https://jbi.global/critical-appraisal-tools (acessado em 08/Mar/2021). 